\title{
Monoclonal Autoantibodies Promote Central Nervous System Repair in an Animal Model of Multiple Sclerosis
}

\author{
David J. Miller,' Kathy S. Sanborn,' Jerry A. Katzmann, ${ }^{2}$ and Moses Rodriguez ${ }^{1,3}$ \\ Departments of 'Immunology, 'Laboratory Medicine and Pathology, and ${ }^{3}$ Neurology, Mayo Clinic and Foundation, \\ Rochester, Minnesota 55905
}

Susceptible strains of mice infected intracerebrally with Theiler's murine encephalomyelitis virus develop a chronic, progressive, immune-mediated CNS demyelinating disease similar both pathologically and clinically to multiple sclerosis. Previous reports indicated that polyclonal immunoglobulins from mice injected with homogenized spinal cord promote CNS remyelination when given to SJL/J mice chronically infected with Theiler's virus. To explore further both the mechanism(s) and potential therapeutic usefulness of antibodies in the treatment of CNS demyelinating diseases, we made a panel of monoclonal antibodies derived from splenocytes of S JL/J mice injected with homogenized spinal cord, and screened them for their autoantigen-binding capability. Monoclonal IgM autoantibodies from two clones, designated SCH94.03 and SCH94.32, promoted fourfold more CNS remyelination than controls when given to chronically infected SJL/J mice. CNS remyelination, assessed morphologically by the presence of abnormally thin myelin sheaths relative to axonal diameter, correlated with the absence of clinical disease progression. In titration experiments, treatment with SCH94.03 and remyelination had a positive dose-response relationship, and as little as $10 \mu \mathrm{g}$ of antibody promoted remyelination. Both SCH94.03 and SCH94.32 showed multiorgan autoreactivity, and recognized both surface and cytoplasmic determinants on glial cells. We propose that this model provides a unique system to elucidate the mechanism(s) and test the reparative potential of autoantibodies in the treatment of CNS injury.

IKey words: Theiler's murine encephalomyelitis virus (TMEV), demyelination, remyelination, immunoglobulin, myelin, oligodendrocyte, glia]

Multiple sclerosis (MS) is a chronic, frequently progressive, inflammatory CNS disease characterized pathologically by primary demyelination, usually without initial axonal injury. The etiology and pathogenesis of MS are unknown. Several immunological features of MS, and its moderate association with certain major histocompatibility complex alleles, have prompt-

Received Jan. 26, 1994; revised Mar. 31, 1994; accepted Apr. 13, 1994

This work was supported by National Multiple Sclerosis Society Grant RG1878-B-2 and National Institutes of Health Grant NS24180. We thank M. Pierce J. Tarara, and T. Kimlinger for their excellent technical assistance, and L. Pease, V. Lennon, D. McCormick, R. Abraham, and B. van Engelen for their helpful comments on the manuscript and on the research. The $5.5 \mathrm{~B} 8$ glial cell line was the generous gift of F. A. McMorris (Wistar Institute, Philadelphia, PA).

Correspondence should be addressed to Dr. Moses Rodriguez, Department of Immunology, Mayo Clinic, 200 First Street SW, Rochester, MN 55905.

Copyright (C) 1994 Society for Neuroscience $0270-6474 / 94 / 146230-09 \$ 05.00 / 0$ ed the speculation that MS is an immune-mediated disease (Hafler and Weiner, 1989; Compston, 1991; Olsson, 1992). An autoimmune hypothesis is supported by the experimental autoimmune (allergic) encephalomyelitis (EAE) model, where injection of certain myelin components into genetically susceptible animals leads to T cell-mediated CNS demyelination (Kabat et al., 1947; Lublin, 1985). However, specific autoantigens and pathogenic myelin-reactive $T$ cells have not been definitively identified in the CNS of MS patients, nor is MS associated with other autoimmune diseases. An alternative hypothesis, based upon epidemiological data (Martyn, 1991), is that an environmental factor, perhaps an unidentified virus, precipitates an inflammatory response in the CNS, which leads to either direct or indirect ("bystander") myelin destruction, potentially with an induced autoimmune component (Lampert, 1978; Rodriguez, 1988). This hypothesis is supported by evidence that several naturally occurring viral infections, in both humans (Rice, 1992) and animals (Dal Canto and Rabinowitz, 1982), can cause demyelination. One commonly utilized experimental viral model is induced by Theiler's murine encephalomyelitis virus (TMEV) (Dal Canto and Lipton, 1977; Rodriguez et al., 1987b). Intracerebral inoculation of genetically susceptible mice with TMEV, a naturally pathogenic enteric picornavirus, produces a chronic, progressive, immune-mediated CNS demyelinating disease, in many aspects indistinguishable from the chronic progressive form of MS. Although some reports have suggested an association of MS with several pathogenic human viruses, no virus to date has been definitively linked with MS (Rice, 1992). The apparently complex etiopathogenesis of MS, potentially involving both environmental and autoimmune factors, may account for the current lack of a precise animal model.

The limited efficacy of current therapies for MS (Goodkin et al., 1992), due primarily to its unknown etiopathogenesis, has stimulated interest in novel therapies to ameliorate this disease (Martin et al., 1992; Steinman, 1992; Weiner et al., 1993). Contrary to the classical view of the humoral immune system playing a pathogenic role in MS, several experimental studies suggest that antibodies $(\mathrm{Ab})$ might play a preventative or reparative role in CNS demyelinating diseases. Injection of CNS components into either guinea pigs with EAE (Traugott et al., 1982), or TMEV-infected mice (Lang et al., 1984), results in improved clinical disease and enhanced CNS remyelination. Although this effect was originally attributed to direct $\mathrm{T}$ cell immunosuppression (Traugott et al., 1982), the hypothesis that injection of myelin components would generate a therapeutic humoral immune response was directly tested in the TMEV model. Immune transfer experiments showed that promotion of remyelination in the TMEV model is mediated by a serum factor (Rodriguez 
et al., 1987a), which was identified as immunoglobulin (Ig) (Rodriguez and Lennon, 1990). Here we report that two monoclonal Abs (mAbs), generated from uninfected mice injected with spinal cord homogenate (SCH) and screened for their autoantigen-binding capability, also promoted CNS remyelination in SJL/J mice chronically infected with TMEV. This model provides the opportunity to investigate the potential beneficial, rather than pathogenic, effect of autoantibodies during the physiological response to tissue injury in the CNS. In addition, elucidation of the mechanism(s) of Ab-mediated CNS remyelination may suggest a rational approach to augment repair in CNS demyelinating diseases.

\section{Materials and Methods}

Virus. We used the Daniel's strain (DA) of TMEV for all experiments. The tissue culture origin and production of this virus have been previously described (Rodriguez et al., 1983).

Animals. SJL/J and BALB/c mice were purchased from the Jackson Laboratory (Bar Harbor, ME); 4-6-week-old SJL/J mice were inoculated intracerebrally with $2 \times 10^{5}$ plaque-forming units (PFU) of TMEV in a $10 \mu$ l volume. MAb treatment was begun 4-6 months after infection, at which time the mice showed clinical signs of chronic demyelination, characterized by ruffled fur, huddled posture, and spasticity, with occasional paralysis and incontinence. Handling of all animals conformed to the National Institutes of Health and Mayo institutional guidelines.

Monoclonal Ab production, screening, and purification. Spleens of two $\mathrm{SJL} / \mathrm{J}$ mice that had been injected twice with SCH in incomplete Freund's adjuvant were used as the source of $B$ cells for fusion and hybridoma production. The serum Igs from these mice had been previously shown to promote remyelination in chronically infected mice (Rodriguez and Lennon, 1990). Splenocytes were fused with NS-1 myeloma cells using polyethyiene glycol, and viable cell fusions were selected with hypoxanthine-aminopterine-thymidine (HAT) media and cloned by limiting dilution as described (Katzmann et al., 1981). Hybridoma supernatants from viable Ig-producing clones were screened for binding to $\mathrm{SCH}$ by an enzyme-linked immunosorbant assay (ELISA); 96-well polystyrene plates (Nunc-Immuno Maxisorp, Nunc Inc., Naperville, IL) were coated with SCH $(10 \mu \mathrm{g} / \mathrm{ml})$ in $100 \mathrm{~mm}$ carbonate buffer, $\mathrm{pH} 8.5$, overnight at $4^{\circ} \mathrm{C}$. Plates were blocked with phosphate-buffered saline (PBS; $100 \mathrm{mM}$ $\mathrm{NaCl}, 50 \mathrm{~mm}$ phosphate, $\mathrm{pH} 7.4$ ) containing $1 \%$ bovine serum albumin (BSA) for $1 \mathrm{hr}$, incubated with mAbs supernatants for $2 \mathrm{hr}$, and washed extensively with PBS plus $0.05 \%$ Tween 20 . Bound Ab was detected with alkaline phosphatase-conjugated goat anti-mouse Ig (Sigma Chemical Co., St Louis, MO) using p-nitrophenyl phosphate as the chromogenic substrate. The hybridomas chosen for treatment experiments were injected into pristane-treated $\mathrm{BALB} / \mathrm{c}$ mice for ascites production. IgM mAbs were purified either by ammonium sulfate precipitation and gel filtration on a Sephacryl S-400 HR (Sigma) column for the initial transfer experiments, or by affinity chromatography using goat antimouse IgM ( $\mu$-chain specific; Jackson Immunoresearch, West Grove, PA) coupled to Reacti-Gel $6 \mathrm{X}$ matrix (Pierce, Rockford. IL) for later transfer experiments.

Treatment protocol and clinical disease assessment. Chronically infected mice were given either intraperitoneal or intravenous injections of $\mathrm{mAb}$ twice weekly for 4-5 weeks. At each treatment injection, mice were assessed clinically by three criteria: appearance, activity, and paralysis. A score for each criterion was given ranging from 0 (no disease) to 3 (severe disease). For appearance, 1 indicated minimal change in coat, 2 indicated a moderate change (scruffy appearance), and 3 indicated a severe change (incontinence and stained coat). For activity, 1 indicated decreased spontaneous movements (minimal ataxia), 2 indicated moderate slowing (minimal spontaneous movements), and 3 indicated severe slowing (no spontaneous movement). For paralysis, 0.5 indicated a spastic extremity, 1 indicated a paralyzed extremity, 1.5 indicated two or more spastic extremities, 2 indicated two paralyzed extremities (unable to walk), 2.5 indicated no righting response, and 3 indicated three or four paralyzed extremities (moribund). The total score for each mouse was the cumulative total from each criterion (maximum of 9). As the clinical score was an ordinal and not a cardinal scale, we used the change in clinical score to assess clinical disease. The clinical assessment data were not disclosed until after the morphological assessment of remyelination was completed.
Light and electron micrograph preparation and assessment of remyelination. Preparation of light and electron microscopy sections and morphological assessment of remyelination were done as previously described (Rodriguez and Lennon, 1990). Briefly, treated mice were anesthetized with pentobarbital $(0.2 \mathrm{mg}$, i.p.), exsanguinated by cardiac puncture, and killed by intracardiac perfusion with Trump's fixative ( $100 \mathrm{~mm}$ phosphate buffer, pH 7.2 , with $4 \%$ formaldehyde and $1.5 \%$ glutaraldehyde). The entire spinal cord was removed carefully from the spinal canal, and sectioned into $1 \mathrm{~mm}$ transverse blocks. Every third block was postfixed in $1 \%$ osmium tetroxide and embedded in Araldite (Polysciences, Warrington, PA). One-micrometer sections from each block were cut and stained with $p$-phenylenediamine. On each section, remyelination was quantitated using a Zeiss interactive digital analysis system (ZIDAS) and camera lucida attached to a Zeiss photomicroscope (Carl Zeiss Inc., Thornwood, NY). Abnormally thin myelin sheaths relative to axonal diameter was used as the criterion for CNS remyelination (Ludwin, 1985, 1988; Fig. $1 A, D$ ). Ten spinal cord sections from each mouse were examined; this corresponded to $8-9 \mathrm{~mm}^{2}$ of white matter examined per mouse. To avoid bias, slides were coded and quantitation was done without knowledge of the treatment groups.

Myelin thickness and axonal diameter measurements and quantitation of myelinated axons. Electron micrographs of normal and remyelinated axons from plastic-embedded spinal cord sections were imaged with a Hamamatsu video camera, digitized, and analyzed using an IBAS 2000 Image Analysis System (Kontron, Munich, Germany). We measured the axonal cross-sectional area with and without the myclin sheath, and used equivalent circle calculations to determine the axonal diameter and myelin sheath thickness. For myelinated axon quantitation, we counted the number of myelinated axons in lesions from plastic-embedded spinal cord sections using the analysis system described above attached to an Axiophot microscope (Carl Zeiss, Inc.). We examined 17 remyelinated and 15 demyelinated lesions in spinal cord sections from animals treated with $\mathrm{mAb} \mathrm{SCH} 94.03$, control IgM, or buffer only; this corresponded to $0.6 \mathrm{~mm}^{2}$ of remyelinated area and $0.8 \mathrm{~mm}^{2}$ of demyelinated area. The criterion for selection of a lesion as demyelinated was the presence of substantial demyelination with minimal repair, whereas remyelinated lesions were chosen based upon the presence of almost complete remyelination throughout the lesion.

TMEV Western blot. Confluent L2 fibroblast cells were infected with TMEV as described (Rodriguez et al., 1983). After $48 \mathrm{hr}$, cells werc solubilized in sodium dodecyl sulfate polyacrylamide gel electrophoresis (SDS-PAGE) sample buffer (62.5 mM Tris, $\mathrm{pH} 6.8,5 \%$ glycerol, $2 \%$ SDS, $14.4 \mathrm{~mm} 2$-mercaptoethanol, $0.02 \%$ bromophenol blue), and proteins were separated by SDS-PAGE on $15 \%$ acrylamide gels and transferred to nitrocellulose by electroblotting. Mock-infected $L 2$ cell lysates were used as a control antigen source to detect $\mathrm{Ab}$ reactivity with nonviral proteins. All electrophoresis and electroblotting were done with the Mini-PROTEAN II apparatus (Bio-Rad, Hercules, CA). Nitrocellulose was air dried for $24 \mathrm{hr}$, rehydrated in water, and blocked with Tris-buffered saline (TBS; $100 \mathrm{~mm} \mathrm{NaCl}, 50 \mathrm{~mm}$ Tris, $\mathrm{pH}$ 7.4) containing $3 \%$ nonfat milk and $0.03 \%$ Tween 20 for $1 \mathrm{hr}$ at room temperature. Nitrocellulose was incubated with control or SCH mAbs $(5 \mu \mathrm{g} / \mathrm{ml})$, polyclonal rabbit anti-TMEV (1:2000; Rodriguez et al., 1983), or serum from chronically infected mice $(1: 200)$, diluted in blocking buffer for 2 hr and washed extensively with TBS containing $0.03 \%$ Tween 20 . Bound $\mathrm{Ab}$ was detected with alkaline phosphatase-labeled goat anti-mouse IgG + IgM or goat anti-rabbit IgG (Jackson Immunoresearch, West Grove, PA) using 5-bromo-4-chloro-3-indolyl phosphate and nitro blue tetrazolium (BCIP/NBT) in $100 \mathrm{~mm} \mathrm{NaCl}, 100 \mathrm{~mm}$ Tris, $5 \mathrm{mM} \mathrm{MgCl}_{2}, \mathrm{pH}$ 9.5 , as the substrate.

In vitro TMEV neutralization assay. Viral plaque assays were done as previously described (Patick et al., 1991). To assess neutralization, aliquots of TMEV (200 PFU/ml) were incubated with various concentrations of $\mathrm{Ab}$ for $1 \mathrm{hr}$ at room temperature prior to plating onto confluent L2 cells. As a positive control, we used serum from susceptible mice chronically infected with TMEV. Under the assay conditions described above, a serum dilution of $1: 34,000$ gave $50 \%$ neutralization, which corresponded to an estimated $20 \mathrm{ng} / \mathrm{ml}$ of TMEV-specific Abs, assuming a total serum Ig concentration of $15 \mathrm{mg} / \mathrm{ml}$, and a TMEVspecific fraction of $5 \%$.

Immunostaining. Rat 5.5B8 glial cells were grown on poly-DL-lysinecoated chamber slides in Dulbecco's modified Eagle's medium (DMEM) supplemented with $1.5 \mathrm{gm} /$ liter D-glucose, $30 \mathrm{nM} \mathrm{SeO}, 15 \mathrm{nM}$ triiodothyronine, $10 \mathrm{ng} / \mathrm{ml}$ biotin, $100 \mu \mathrm{M} \mathrm{ZnCl}_{2}, 50 \mu \mathrm{g} / \mathrm{ml}$ gentamicin, and $10 \%$ fetal bovine serum. All staining steps were done at room temper- 

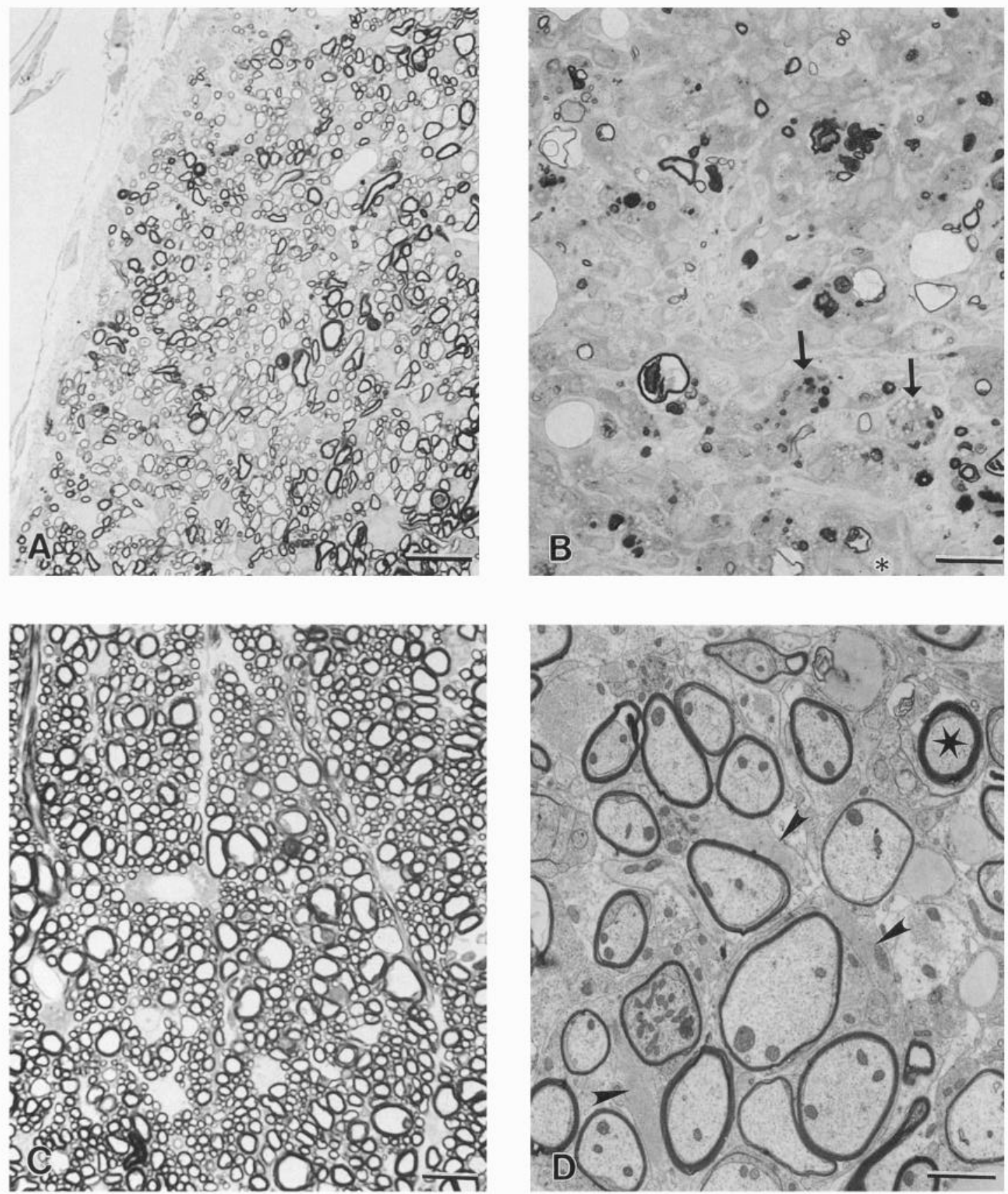

Figure 1. Light and electron micrographs of CNS remyelination promoted by mAb SCH94.03. $A$, Light micrograph of spinal cord section from a chronically infected $\mathrm{SJL} / \mathrm{J}$ mouse treated with $\mathrm{SCH} 94.03$ showing CNS remyelination. $B$, Light micrograph of spinal cord section from a chronically infected SJL/J mouse treated with a control IgM showing extensive demyelination, and the relative absence of remyelination. There are numerous inflammatory cells, including macrophages with ingested myelin debris (arrows). The asterisk indicates a representative naked axon, many of which are present throughout the lesion. $C$, Light micrograph of spinal cord section with normal myelin. $D$, Electron micrograph of spinal cord section from an animal treated with SCH94.03 showing multiple axons with abnormally thin myelin sheaths relative to axon diameter. The star to the upper right indicates an axon with normal myelin sheath thickness. Arrowheads point to astrocytic processes, which are intimately associated with remyelinated axons. Scale bars: $A-C, 13 \mu \mathrm{m} ; D, 2 \mu \mathrm{m}$.

ature. For surface staining, slides were briefly rinsed with PBS, and cells were lightly fixed with $1 \%$ formaldehyde in PBS for $10 \mathrm{~min}$ to prevent cell detachment during subsequent staining steps. For cytoplasmic staining, slides were rinsed twice in PBS and either air dried for $1 \mathrm{hr}$ or incubated with $0.1 \%$ Triton X-100 in PBS for 10 min. Cells were blocked in $2 \%$ BSA for $30 \mathrm{~min}$, washed, incubated with control IgM or mAb $\mathrm{SCH} 94.03(10 \mu \mathrm{g} / \mathrm{ml}$ in $1 \% \mathrm{BSA})$ for $1 \mathrm{hr}$, and washed extensively with PBS. After fixation with $4 \%$ paraformaldehyde for 15 min, slides were incubated with fluorescein-labeled goat anti-mouse IgM (Jackson Im- munoresearch) for $1 \mathrm{hr}$, washed with PBS, coverslipped with $10 \%$ MOWIOL (Hoechst) in $100 \mathrm{~mm}$ Tris, $25 \%$ glycerol, $\mathrm{pH} 8.5$, with 25 $\mu \mathrm{g} / \mathrm{ml}$ 1,4-diazobicyclo-[2.2.2]-octane (DABCO) to prevent fading, and allowed to set overnight in the dark. For frozen tissue sections, fresh neonatal rat, adult mouse, or autopsied human cortical brain tissue was quick frozen in isopentane chilled in liquid nitrogen prior to liquid nitrogen storage. Frozen sections $(10 \mu \mathrm{m})$ were transferred onto gelatinized glass microscope slides, air dried for $4-8 \mathrm{hr}$, and stored at $-70^{\circ} \mathrm{C}$. Prior to immunostaining, slides were placed at room temperature over- 
night. The immunoperoxidase staining protocol was similar that dcscribed above, using the $\mathrm{ABC}$ immunoperoxidase reagent (Vector Laboratories, Burlingame, CA), developed with $1.5 \mathrm{mg} / \mathrm{ml}$ Hanker-Yates reagent ( $p$-phenylene diamine-procatechol) in $50 \mathrm{~mm}$ Tris, $\mathrm{pH} 7.6$, with $0.034 \% \mathrm{H}_{2} \mathrm{O}_{2}$, counterstained with Mayer's hematoxylin, and mounted with Permount (Fischer Scientific, Pittsburgh, PA).

Data analysis. We used a modified cumulative rank sum test (O'Brien, 1984) to compare remyelination between treatment groups. This statistical test takes into account several numerically unrelated parameters of therapeutic effectiveness, and is used routinely for clinical trial efficacy assessment. Parallel analyses using a standard unpaired Student's $t$ test to compare individual parameters of remyelination gave equivalent results. Comparisons of disease severity and correlation significance were determined by a one-way analysis of variance (ANOVA). Statistical analyses were done with the either the SIGMASTAT (Jandel Scientific, San Rafael, CA) or EXCEL (Microsoft Corporation, Redmond, WA) software programs. Calculated values were considered significant when $p$ was $<$ 0.05 .

\section{Results}

Selection of $S C H$ mAbs to promote CNS remyelination

We constructed a panel of $\mathrm{mAbs}$ derived from splenocytes of uninfected SJL/J mice injected with SCH, whose serum Igs were shown previously to promote remyelination in vivo (Rodriguez and Lennon, 1990). After the initial fusion and cloning, 2 of the 95 wells with viable Ig-secreting hybridomas contained Ab with significant binding to SCH by ELISA. Hybridoma cells from these two wells, called the 79 and 94 series, were subcloned by limiting dilution and screened again for binding to SCH by ELISA. For the 79 series hybridomas, 14 of 49 clones were positive by SCH ELISA, while for the 94 series, 17 of 32 were positive for binding to SCH. Based upon the ELISA data, we chose two 79 series hybridomas (SCH79.08 and SCH79.27), both of which also reacted with myelin basic protein (MBP) by ELISA, and three 94 series hybridomas (SCH94.03, SCH94.11, and SCH94.32), none of which reacted with MBP, for ascites production and in vivo transfer experiments. Initial treatment experiments showed that $\mathrm{mAbs}$ from SCH94.03 and SCH94.32, both of which were an IgM isotype, promoted significantly more CNS remyelination in mice chronically infected with TMEV than the other three hybridomas, and therefore were chosen for a more detailed examination of their potential to promote $\mathrm{CNS}$ repair.

\section{CNS remyelination promoted by mAbs SCH94.03 and SCH94.32}

SJL/J mice chronically infected with TMEV and treated with either mAb SCH94.03 or SCH94.32 showed significantly greater CNS remyelination than animals treated with either isotypematched control Ab or buffer only (Table 1). Remyelination was seen with either intravenous or intraperitoneal injections (data pooled). SCH94.03- or SCH94.32-treated animals had approximately two- to threefold more remyelinated lesions, and a threeto fourfold larger total area of CNS remyelination than control animals. When a cumulative statistical comparison was made using these two parameters of therapeutic effectiveness, the CNS remyelination induced by $\mathrm{mAbs} \mathrm{SCH} 94.03$ and $\mathrm{SCH} 94.32$ was highly significant ( $p<0.005$; Table 1$)$. In a chronic progressive disease like TMEV infection, the extent of CNS repair is a direct function of the extent of CNS damage. Both the number and area of CNS lesions were not different between treatment groups, indicating similar disease severity (Table 1). When CNS remyelination was expressed as the percentage of lesion area showing remyelination, approximately one-third of the cumulative demyelinated lesion area showed CNS remyelination in mice treated with either $\mathrm{mAb}$ SCH94.03 or SCH94.32 (Table 1). The extent of remyelination was comparable to that observed previously in mice treated with SCH antiserum (Rodriguez et al., 1987a) or SCH IgG (Rodriguez and Lennon, 1990).

\section{Morphology of CNS remyelination}

CNS remyelination was readily identified morphologically both by light and electron microscopy (Fig. 1). Figure $1 A$ shows a remyelinated lesion from an animal treated with SCH94.03. The majority of axons in the lesion show morphologic evidence of repair, with abnormally thin myelin sheaths relative to axonal diameter (Ludwin, 1985, 1988). For comparison, Figure $1 B$ shows a demyelinated lesion, with minimal remyelination, whereas Figure $1 C$ is an area of normal myelin, with thickly myelinated axons. Within remyelinated lesions (Fig. $1 A$ ), there were $15.3 \pm 1.0\left(\right.$ mean \pm SEM) myelinated axons per $100 \mu \mathrm{m}^{2}$, compared to only $1.1 \pm 0.2$ myelinated axons per $100 \mu \mathrm{m}^{2}$ in demyelinated lesions (Fig. $1 B$ ). By electron microscopy, CNS remyelination was especially evident (Fig. $1 D$ ). Almost every axon in the field has evidence of new myelin formation, although the degree of remyelination (i.e., myelin thickness) is variable between individual axons, suggesting different stages of the repair process. The ratio of myelin thickness to axonal diameter was $0.08 \pm 0.01$ (mean $\pm \mathrm{SEM} ; n=25$ axons) for remyelinated axons compared to $0.21 \pm 0.01(n=34$ axons) for normally myelinated axons.

\section{Correlation between clinical disease and morphological remyelination}

We assessed whether morphological remyelination correlated with clinical signs of disease improvement. At each treatment injection, mice were assessed clinically as described in Materials and Methods. We correlated the changc in clinical score with the percentage of lesion area showing remyelination (Fig. 2). A positive change in clinical score indicates worsening of disease. Using data from all treatment groups, the change in clinical score showed a moderate but significant negative correlation $(R$ $=-0.40 ; p<0.04$ ) with the percentage of lesion area showing remyelination. Although few animals actually improved clinically ( $\Delta$ clinical score $<0$ ), animals with an increase in disease severity $(\Delta$ clinical score $>0$ ) tended to have less morphological remyelination, while animals that remained stable clinically $(\Delta$ clinical score $=0$ ) showed the most remyelination. A similar negative correlation was obtained when we used the other quantitative measures of remyelination, the number of remyelinated lesions and the area of remyelination, as shown in Table 1. We conclude that remyelination quantitated by morphology is associated with slowing of clinical disease progression.

\section{Titration of mAb SCH94.03 dose and CNS remyelination}

For the initial treatment experiments, we empirically chose a total $\mathrm{mAb}$ dose of $25 \mathrm{mg} / \mathrm{kg}$ for intravenous injections and 250 $\mathrm{mg} / \mathrm{kg}$ for intraperitoneal injections. To assess the dose-response characteristics, and to determine the minimal amount of $\mathrm{mAb}$ needed to promote remyelination, we treated chronically infected mice with various intraperitoneal doses of SCH94.03. Both the number of remyelinated lesions (data not shown) and the total area of remyelination (Fig. 3) increased significantly with larger doses of $\mathrm{SCH} 94.03$. The positive correlation between the dose of $\mathrm{mAb} \mathrm{SCH} 94.03$ and CNS remyelination was especially striking when the severity of CNS disease was taken into account. When CNS repair was expressed as the 
Figure 2. Correlation between the change in clinical disease and morphological remyelination. Morphological remyelination is represented as the percentage of lesion area showing CNS remyelination. A change in clinical score of 0 represents stable disease over the treatment period (4-5 weeks), whereas a positive change indicates worsening of clinical disease, and a negative change indicates improvement. Data represent individual animals from all treatment groups.

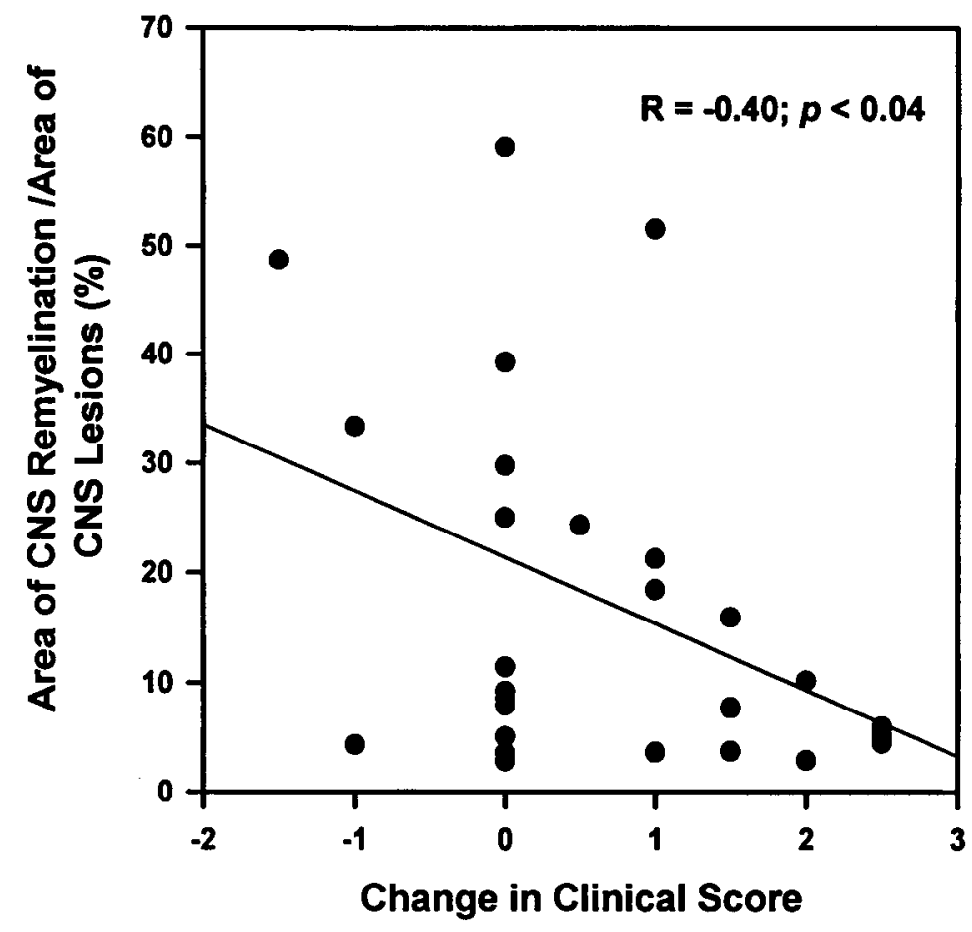

rabbits injected with purified TMEV (Rodriguez et al., 1983). In addition, we observed no significant inhibition of TMEV infectivity in vitro with up to $5 \mu \mathrm{g} / \mathrm{ml}$ of either $\mathrm{SCH} 94.03$ or $\mathrm{SCH} 94.32$, under assay conditions where $50 \%$ neutralization was observed with a 1:34,000 dilution of serum from chronically infected animals. These results indicated that the therapeutic effect of SCH94.03 and SCH94.32 was not due to direct inhibition of the virus. This is consistent with the observation that chronically infected animals that show little spontaneous remyelination maintain high endogenous neutralizing $\mathrm{Ab}$ titers (Patick et al., 1991).

To characterize initially the autoantigens recognized by mAbs SCH94.03 and SCH94.32, we immunostained various cell lines derived from glial (rat C6, mouse G26-20, human U373MG and U87MG), neural (human neuroblastoma), fibroblast (mouse L and 3T3), epithelial (human SCC-9 carcinoma), and lymphocytic (mouse CTLL2) origin. Both mAbs stained internal antigens of all cell lines tested, which indicated that certain antigens

did not react with any TMEV proteins recognized by either serum from chronically infected mice or polyclonal IgG from

Table 1. Monoclonal antibodies SCH94.03 and SCH94.32 promote CNS remyelination

\begin{tabular}{|c|c|c|c|c|c|c|c|}
\hline Treatment & $n$ & $\begin{array}{l}\text { Number of } \\
\text { remyelinated } \\
\text { lesions }\end{array}$ & $\begin{array}{l}\text { Area of } \\
\text { remyelination } \\
\left(\mathrm{mm}^{2}\right)\end{array}$ & $p$ & $\begin{array}{l}\text { Number of } \\
\text { demyelinated } \\
\text { lesions }\end{array}$ & $\begin{array}{l}\text { Area of } \\
\text { lesion }\left(\mathrm{mm}^{2}\right)\end{array}$ & $\begin{array}{l}\text { Area remyelination/ } \\
\text { area lesion }(\%)\end{array}$ \\
\hline SCH94.03 & 12 & $12.8 \pm 2.6$ & $0.35 \pm 0.09$ & $<0.0025$ & $25.8 \pm 2.6$ & $1.09 \pm 0.19$ & $28.9 \pm 3.8$ \\
\hline SCH 94.32 & 12 & $13.3 \pm 2.3$ & $0.42 \pm 0.11$ & $<0.0001$ & $24.9 \pm 2.8$ & $1.46 \pm 0.21$ & $26.7 \pm 4.2$ \\
\hline IgM control & 13 & $6.7 \pm 1.2$ & $0.11 \pm 0.02$ & - & $29.9 \pm 2.0$ & $1.70 \pm 0.28$ & $7.7 \pm 1.8$ \\
\hline Buffer only & 11 & $5.3 \pm 1.1$ & $0.06 \pm 0.01$ & - & $27.7 \pm 2.7$ & $1.11 \pm 0.29$ & $6.5 \pm 1.2$ \\
\hline
\end{tabular}

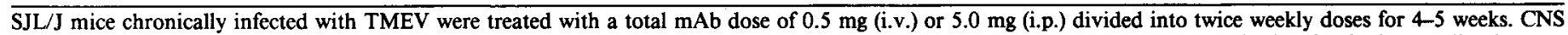

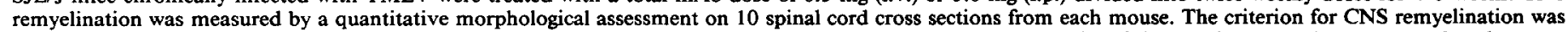

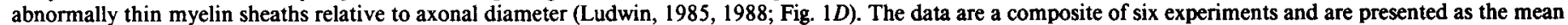

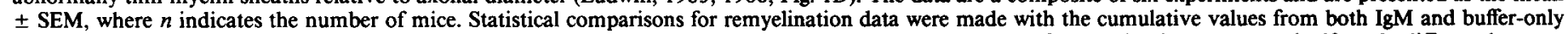

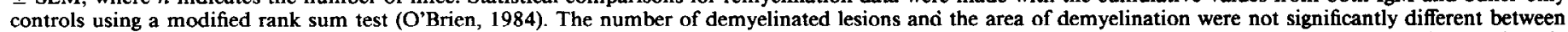

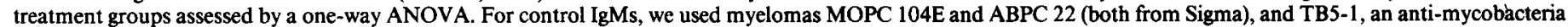
$\mathrm{mAb}$. 


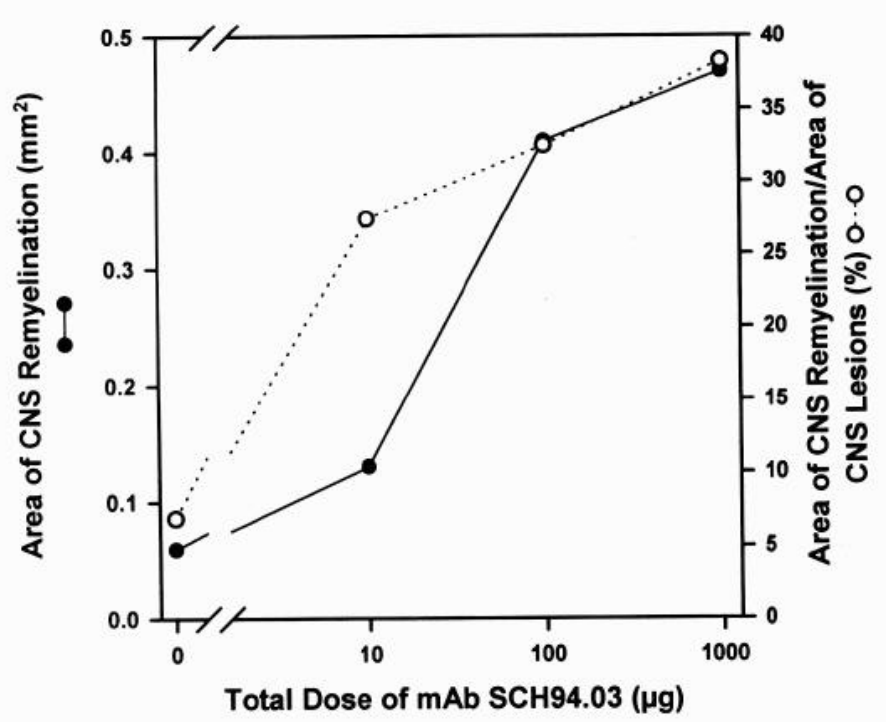

Figure 3. Dose-response relationship between treatment with $\mathrm{mAb}$ SCH94.03 and CNS remyelination: area of CNS remyelination $(\bullet)$ and percentage of lesion area with remyelination $(O)$ in animals treated with various doses of mAb SCH94.03. Remyelination was quantitated as described in Table 1. Data are the mean values of four or five animals per $\mathrm{mAb}$ dose, with the final cumulative dose indicated on the graph; SEM averaged $35 \%$ of the mean. There was no statistical difference assessed by one-way ANOVA in the number of demyelinated lesions or the area of demyelination between treatment groups, indicating similar extent of disease in all animals. The number of demyelinated lesions and area of lesions were $33.2 \pm 7.5$ and $1.25 \pm 0.43$ for the $1000 \mu \mathrm{g}$ group, $31.8 \pm 8$ and $1.11 \pm 0.31$ for the $100 \mu \mathrm{g}$ group, $23.8 \pm 3.4$ and $0.54 \pm 0.14$ for the $10 \mu \mathrm{g}$ group, and $29.0 \pm 6.5$ and $0.74 \pm 0.20$ for the buffer only group (represented as the 0 dose point on the graph). Animals treated with $100 \mu \mathrm{g}$ of control IgM (MOPC 104E) had remyelination scores similar to control animals treated with buffer only.

recognized by these mAbs were not restricted to unique cell types in vitro. Based on the hypothesis that the therapeutic effect of SCH94.03 and SCH94.32 was due to a CNS-specific interaction, we further investigated the immunostaining of cultured cells by $\mathrm{SCH} 94.03$ and $\mathrm{SCH} 94.32$ using the rat glial cell line 5.5B8 (Fig. 5A,B). This immortalized glial cell line has phenotypic characteristics of both oligodendrocytes and astrocytes, with expression of MBP and 2',3'-cyclic nucleotide $3^{\prime}$-phosphodiesterase (CNP), and low but detectable expression of glial fibrillary acidic protein (GFAP) and the lipids or proteins recognized by the mAbs A2B5 and O4 (Bozyczko et al., 1990). SCH94.03 and SCH94.32 recognized both a surface and cytoplasmic determinant on 5.5B8 cells. The surface staining was most prominent on small cells that lay on top of a layer of flat, morphologically differentiated cells (Fig. $5 A$ ). Surface staining was confirmed by flow cytometry on live cells. When the cell membrane was permeabilized by dehydration or brief treatment with a nonionic detergent to expose internal antigens, the staining pattern was altered considerably (Fig. $5 B$ ). The cytoplasmic staining was filamentous, with a dense perinuclear network that extended out into the cell processes. This pattern closely resembled the staining pattern of the intermediate filament cytoskeletal protein vimentin. These data indicated that $\mathrm{SCH} 94.03$ and SCH94.32 recognized antigens that were not restricted to cells derived from the nervous system, but that they did recognize both surface and cytoplasmic determinants on glial cells.

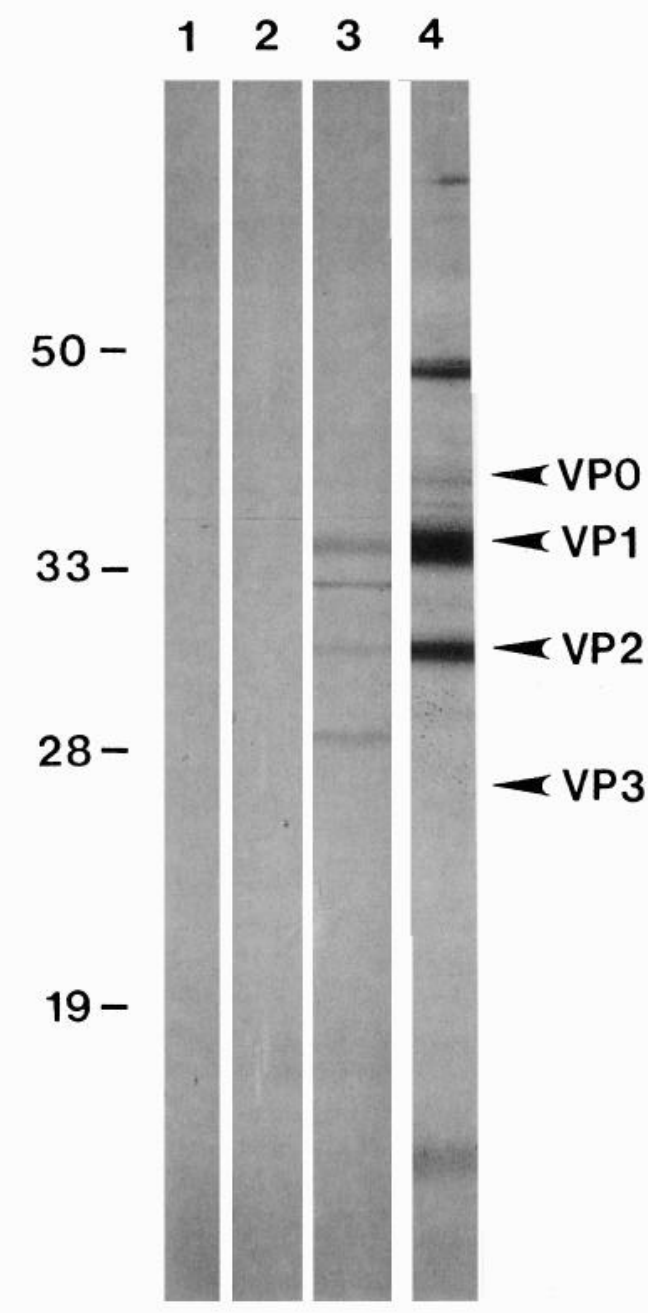

Figure 4. Western blot of TMEV proteins. Lysates from infected L2 fibroblast cells were separated by SDS-PAGE, transferred to nitrocellulose, and blotted with SCH94.03 (lane 1), SCH94.32 (lane 2), serum from susceptible mice chronically infected with TMEV (lane 3), and polyclonal rabbit anti-TMEV IgG (lane 4). Molecular weights are indicated on the left in kilodaltons. The position and identification of the major TMEV capsid proteins are indicated on the right. Western blot of lysates from control mock infected L2 cells showed single bands with the serum from chronically infected animals and the polyclonal rabbit anti-TMEV IgG at 32 and $43 \mathrm{kDa}$, respectively, but no reactivity with SCH94.03 or $\mathrm{SCH} 94.32$.

Immunohistochemical staining of frozen mouse, rat, and human tissue confirmed that $\mathrm{SCH} 94.03$ and $\mathrm{SCH} 94.32$ were not CNS-specific mAbs, but rather showed multiorgan reactivity. Both mAbs immunostained all major organs examined, including the brain, spinal cord, heart, liver, kidney, stomach, and small intestine. However, not all cells within an organ stained, suggesting in situ cytological specificity (D. Miller and M. Rodriguez, unpublished observations). Within the CNS, SCH94.03 and $\mathrm{SCH} 94.32$ stained predominantly blood vessels, ependymal cells, and stellate-shaped cells with the morphological features of glial cells, which were enriched in neonatal cerebellar, periventricular, and brainstem white matter (Fig. $5 C$ ), and both neonatal and adult optic nerve. Similar glial cells positive for SCH94.03 and SCH94.32 were found in autopsied human brain tissue, especially at the gray-white matter junction (Fig. $5 D$ ). 

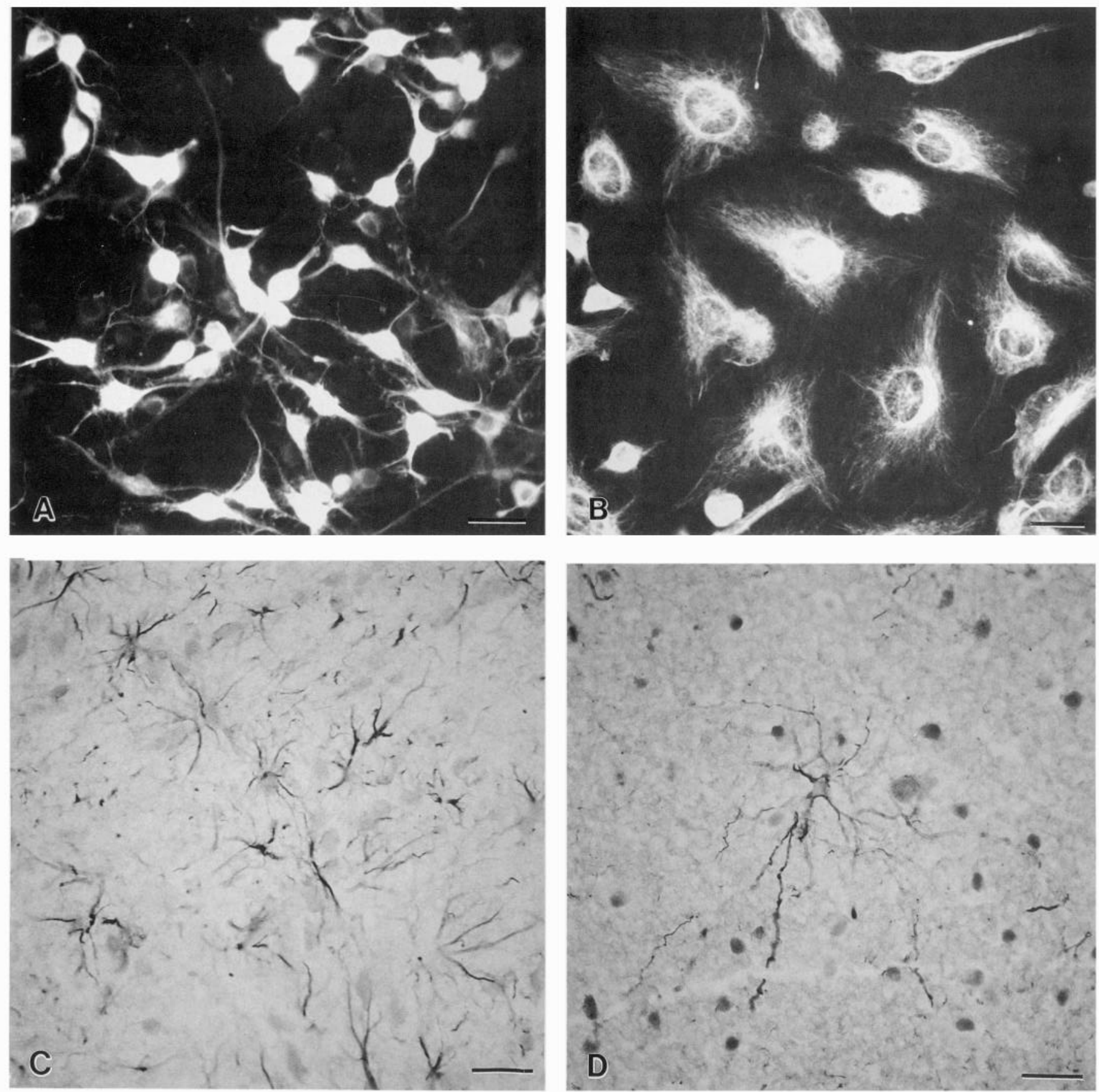

Figure 5. Immunostaining of cultured glial cells and frozen CNS tissue sections with mAb SCH94.03. $A$ and $B$, Immunofluorescence surface staining $(A)$ of 5.5B8 glial cells is restricted to small cells that lie on top of the culture, while the cytoplasmic staining $(B)$ is filamentous, suggestive of cytoskeletal elements. $C$ and $D$, Immunoperoxidase staining of frozen CNS tissue sections show stellate-shaped glial cells prominent in neonatal rat brainstem $(C)$ and in autopsied human cortical brain at the gray-white matter junction $(D)$. Identical immunostaining results were obtained with mAb SCH94.32. Immunostaining with a control IgM (MOPC 104E) was negative for all samples and tissue structures that immunostained with $\mathrm{SCH} 94.03$ and $\mathrm{SCH} 94.32$. Scale bars, $15 \mu \mathrm{m}$.

\section{Discussion}

Previous work from our laboratory has shown that treatment of chronically infected $\mathrm{SJL} / \mathrm{J}$ mice with polyclonal $\mathrm{IgG}$ directed against unknown components of $\mathrm{SCH}$ stimulates CNS remyelination by oligodendrocytes (Rodriguez and Lennon, 1990). In this report, we expand this observation and demonstrate that two mAbs, generated and screened for their autoantigen-binding capability, also promoted CNS remyelination. Mice chronically infected with TMEV and treated either intravenously or intraperitoneally with IgM mAbs from hybridomas $\mathrm{SCH} 94.03$ or SCH94.32 had significantly more CNS repair than control animals, measured by a detailed quantitative morphological assessment of CNS remyelination.

We propose two potential mechanisms by which Abs promote remyelination. First, Abs might inhibit some pathogenic com- 
ponent of the disease process, such as virus activity, an immune response that directly induces demyelination, or an immune response that prevents remyelination. If the disease outcome is based upon a balance between tissue destruction and repair, inhibition of pathogenic components would allow a physiological repair response to predominate. Experimental and clinical evidence support this hypothesis. Spontaneous CNS remyelination is seen in MS patients and several experimental models of CNS demyelination (Ludwin, 1988). We also observed spontaneous remyclination in control mice (Table 1). This indicates that remyelination is a normal physiological response to myelin damage. In addition, treatment of mice chronically infected with TMEV with various immunosuppressive regiments promotes remyelination, but does not decrease demyelination, indicating that there is an immunological component that inhibits remyelination (Rodriguez and Lindsley, 1992). Preliminary immunological function studies have indicated that animals treated with SCH94.03 had similar numbers of B and T (both CD4+ and $\mathrm{CD}^{+}$) cells in their spleens compared to control animals, had similar in vitro splenocyte proliferative responses to $\mathrm{mi}$ togens and antigens, and mounted comparable $\mathrm{Ab}$ responses to both $\mathrm{T}$ cell-dependent and $\mathrm{T}$ cell-independent antigens (Miller and Rodriguez, unpublished observations). We are currently investigating the possibility that these mAbs induce a subtle immunomodulatory effect in chronically infected animals, similar to mechanisms proposed for the therapeutic effect of polyclonal Ig in certain human inflammatory diseases (reviewed by Dwyer, 1992).

The second hypothesis is that certain Abs can actively stimulate CNS remyelination, perhaps via stimulation of oligodendrocyte proliferation and/or differentiation in vivo, as has been demonstrated in vitro (Diaz et al., 1978; Lehrer et al., 1979; Raine et al., 1979; Bansal et al., 1988; Benjamins and Dyer, 1990; Dyer, 1993). MAbs SCH94.03 and SCH94.32 may directly stimulate precursor oligodendrocytes, which are known to be present at the edges of both human (Raine et al., 1981; Prineas, 1985) and experimental (Moore et al., 1984; Rodriguez and Lennon, 1990) CNS lesions that show active remyelination. Alternatively, SCH94.03 and SCH94.32 may work indirectly, via activation of astrocytes or other accessory cells, which could release factors important for the survival or proliferation of cells in the oligodendroglial lincagc. The formation of $\mathrm{Ab}$-antigen complexes in situ with tissue components released upon myelin destruction may also participate in Ab-mediated CNS remyelination. Although SCH94.03 and SCH94.32 were not CNS specific, the recognition of both surface and cytoplasmic antigens on glial cells by both mAbs (Fig. 5) supports an active mechanism hypothesis. In contrast to the immunomodulatory hypothesis, which would not necessarily require that Abs have direct access to the CNS, the hypothesis that Abs actively stimulate CNS remyelination implies the prerequisite of direct access to the CNS. This is contrary to the view of the selective permeability of the blood-brain barrier, especially toward large molecules such as pentameric IgM. However, during chronic inflammatory conditions such as TMEV infection or MS, peripheral leukocytes migrate into the CNS, indicating an alteration in the blood-brain barrier permeability. Therefore, large protcins such as scrum Ig might also enter, via either passive diffusion through "open" endothelium, or perhaps via an unidentified active transport mechanism. We are currently assessing whether SCH94.03 and SCH94.32 can enter the CNS, and whether either $\mathrm{mAb}$ can directly stimulate glial cells in vitro.
Definitive identification of the antigens recognized by SCH94.03 and SCH94.32 may provide some clue as to a mechanism of Ab-mediated remyelination. Our objective in the screening and selection of these mAbs was not to select for reactivity toward a unique antigen, but rather to select broadly for reactivity toward a heterogeneous mixture enriched for CNS antigens ( $\mathrm{SCH}$ ), and subsequently screen for therapeutic biological activity. Our identification of two mAbs that promoted remyelination, but were not CNS specific, was unanticipated, but not surprising. In a previous study, 19 of 30 hybridomas made using brain tissue as the immunogen reacted with both neural and non-neural tissue (Franko et al., 1981). Nineteen of these hybridomas secreted an IgM mAb, five reacted with multiple cell lines derived from different tissues, and several had immunostaining patterns suggestive of cytoskeletal proteins, similar to our observations with SCH94.03 and SCH94.32 (Fig. 5). Our identification of two IgM monoclonal autoantibodies with multiorgan reactivity, and recognition of both surface and cytoplasmic (presumably cytoskeletal) antigens, suggests that SCH94.03 and SCH94.32 may belong to a family of antibodies known as natural autoantibodies. Natural autoantibodies, whose physiological significance is unknown, are usually IgMs, are present in the serum of normal individuals, represent a substantial fraction of the total Ig repertoire, show polyreactivity toward multiple self and nonself antigens, including cytoskeletal elements, and are typically encoded by unmutated germline Ig genes (Avrameas and Ternynck, 1993). We are directly investigating whether SCH94.03 and SCH94.32 have similar phenotypic characteristics.

In CNS demyelinating diseases, Abs are generally considered pathogenic. The initial observation that polyclonal Igs promote CNS remyelination in an animal model of MS (Rodriguez and Lennon, 1990), and our similar results with two mAbs presented in this report, questions the traditional role of autoantibodies as pathogenic, and should stimulate interest in the potentially beneficial role of autoantibodies in normal physiology. In addition, the identification of $m A b s$ that facilitate tissue repair represents a significant step toward elucidation of the mechanism(s) involved in Ab-mediated CNS repair.

\section{References}

Avrameas S, Ternynck T (1993) The natural autoantibodies system: between hypotheses and facts. Mol Immunol 30:1133-1142.

Bansal R, Gard AL, Pfeiffer SE (1988) Stimulation of oligodendrocyte differentiation in culture by growth in the presence of a monoclonal antibody to sulfated glycolipid. J Neurosci Res 21:260-267.

Benjamins JA, Dyer CA (1990) Glycolipid and transmembrane signaling in oligodendroglia. Ann NY Acad Sci 605:90-100.

Bozyczko D, deFerra F, McMorris FA (1990) Production of immortalized cells of the oligodendroglial lineage. Ann NY Acad Sci 605: 350-353.

Compston DAS (1991) Genetic susceptibility to multiple sclerosis. In: McAlpine's multiple sclerosis (Matthews B, ed), pp 301-319. London: Churchil Livingstone.

Dal Canto MC, Lipton HL (1977) Multiple sclerosis: animal model. Theiler's virus infection in mice. Am J Pathol 88:497-500.

Dal Canto MC, Rabinowitz SG (1982) Experimental models of virusinduced demyelination in the central nervous system. Ann Neurol 11:109-127.

Diaz M, Bornstein MB, Raine CS (1978) Disorganization of myelinogenesis in tissue culture by anti-CNS antiserum. Brain Res 154: 231-239.

Dwyer JM (1992) Drug therapy: manipulating the immune system with immune globulin. N Engl J Med 326:107-116.

Dyer CA (1993) Novel oligodendrocyte transmembrane signaling sys- 
tems. Investigations utilizing antibodies as ligands. Mol Neurobiol $7: 1-22$.

Franko MC, Masters CL, Gibbs CJ Jr, Gajdusek DC (1981) Monoclonal antibodies to central nervous system antigens. J Neuroimmunol 1:391-411.

Goodkin DE, Ransohoff RM, Rudick RA (1992) Experimental therapies for multiple sclerosis: current status. Clev Clin J Med 59:6374.

Hafler DA, Weiner HL (1989) MS: a CNS and systemic autoimmune disease. Immunol Today 10:104-107.

Kabat EA, Wolf A, Bezer AE (1947) The rapid production of acute disseminated encephalomyelitis in rhesus monkeys by injection of heterologous and homologous brain tissue with adjuvants. J Exp Med 85:117-129.

Katzmann JA, Nesheim ME, Hibbard LS, Mann KG (1981) Isolation of functional human coagulation factor $\mathrm{V}$ using a hybridoma antibody. Proc Natl Acad Sci USA 78:162-166.

Lampert PW (1978) Autoimmune and virus-induced demyelinating diseases: a review. Am J Pathol 91:176-208.

Lang W, Rodriguez M, Lennon VA, Lampert PW (1984) Demyelination and remyelination in murine viral encephalomyelitis. Ann NY Acad Sci 436:98-102.

Lehrer GM, Maker S, Silides DJ, Weiss C, Bornstein MB (1979) Stimulation of myelin lipid synthesis in vitro by white matter antiserum in the absence of complement. Brain Res 172:557-560.

Lublin FD (1985) Relapsing experimental allergic encephalomyelitis: an autoimmune model of multiple sclerosis. Spinger Semin Immunopathol 8:197-208.

Ludwin SK (1985) Remyelination in the central nervous system of the mouse. In: The Pathology of the myelinated axon (Adachi $M$, Hirano A, Aronson SM, eds), pp 49-79. Tokyo: Igaku-Shoin.

Ludwin SK (1988) Remyelination in the central nervous system and the peripheral nervous system. Adv Neurol 47:215-254.

Martin R, McFarland HF, McFarlin DE (1992) Immunological aspects of demyelinating diseases. Annu Rev Immunol 10:153-187.

Martyn C (1991) The epidemiology of multiple sclerosis. In: McAlpine's multiple sclerosis (Matthews B, ed), pp 3-40. London: Churchil Livingstone.

Moore GRW, Traugott U, Raine CS (1984) Survival of oligodendrocytes in chronic relapsing experimental autoimmune encephalomyelitis. J Neurol Sci 65:137-145.

O'Brien PC (1984) Procedures for comparing samples with multiple endpoints. Biometrics 40:1079-1087.
Olsson T (1992) Immunology of multiple sclerosis. Curr Opin Neurol Neurosurg 5:195-202.

Patick AK, Thiemann RL, O'Brien PC, Rodriguez M (1991) Persistence of Theiler's virus infection following promotion of central nervous system remyelination. J Neuropathol Exp Neurol 50:523-537.

Prineas JW (1985) The neuropathology of multiple sclerosis. In: Handbook of clinical neurology, Vol 3, Demyelinating diseases (Pinker PJ, Bruyn GW, Klawans HL, eds), pp 213-257. Amsterdam: Elsevier.

Raine CS, Diaz M, Pakingan M, Bornstein MB (1979) Antiseruminduced dissociation of myelinogenesis in vitro: an ultrastructural study. Lab Invest 38:397-403.

Raine CS, Scheinberg L, Waltz JM (1981) Multiple sclerosis: oligodendrocyte survival and proliferation in an active lesion. Lab Invest 45:534-546.

Rice GPA (1992) Virus-induced demyelination in man: models for multiple sclerosis. Curr Opin Neurol Neurosurg 5:188-194.

Rodriguez M (1988) Mechanisms of virus-induced demyelination and remyelination. Ann NY Acad Sci 540:240-251.

Rodriguez M, Lennon VA (1990) Immunoglobulins promote remyelination in the central nervous system. Ann Neurol 27:12-17.

Rodriguez. M, Lindsley MD (1992) Immunosuppression promotes CNS remyelination in chronic virus-induced demyelinating disease. Neurology 42:348-357.

Rodriguez M, Leibowitz JL, Lampert PW (1983) Persistent infection of oligodendrocytes in Theiler's virus-induced encephalomyelitis. Ann Neurol 13:426-433.

Rodriguez M, Lennon VA, Benveniste EN, Merrill JE (1987a) Remyelination by oligodendrocytes stimulated by antiserum to spinal cord. J Neuropathol Exp Neurol 46:84-95.

Rodriguez M, Oleszak E, Leibowitz J (1987b) Theiler's murine encephalomyelitis virus: a model of demyelination and viral persistence. CRC Crit Rev Immunol 7:325-365.

Steinman L (1992) The development of rational strategies for selective immunotherapy against autoimmune demyelinating diseases. Adv Immunol 49:357-379.

Traugott U, Stone SH, Raine CS (1982) Chronic relapsing experimental autoimmune encephalitis treatment with combinations of myelin components promotes clinical and structural recovery. J Neurol Sci 56:65-73.

Weiner HL, Mackin GA, Matsui M, Orav EJ, Khoury SJ, Dawson DM, Hafler DA (1993) Double-blind pilot trial of oral tolerization with myelin antigens in multiple sclerosis. Science 259:1321-1324. 